\section{AB0084 INCREASED LEVEL OF NEUROPILIN-1 IN CD4+ T CELL AND ITS CORRELATION WITH DISEASE ACTIVITY OF SYSTEMIC LUPUS ERYTHEMATOSUS}

Y. J. Choi ${ }^{1}$, E. K. Lee ${ }^{1}$, M. S. Lee ${ }^{2}$, C. H. Lee ${ }^{2}$, C. H. Chung ${ }^{2}$, W. H. Yoo ${ }^{1}$. ${ }^{1}$ Research Institute of Clinical Medicine of Jeonbuk National UniversityBiomedical Research Institute of Jeonbuk National University Hospital, Internal Medicine, Jeonju, Korea, Rep. of (South Korea); ${ }^{2}$ Wonkwang University Hospital, Internal Medicine, Iksan, Korea, Rep. of (South Korea)

Background: Semaphorin has been found as a neuronal guidance molecule, but has recently been called "immune semaphorin", as their critical role in immune cell activation, differentiation and migration has been revealed. In particular, class 4 semaphorin has been shown to contribute to lymphocyte activation and immune homeostasis.

Objectives: This study was aimed to investigate the expression of neuropilin-1 (NRP-1), the receptor of class 4 semaphorin, in the murine mouse model of systemic lupus erythematosus (SLE) and the patients with SLE and the correlation between the expression of NRP-1 and disease activity of SLE.

Methods: The expression of NRP-1 was measured in T cells in spleen and renal tissue in control mouse and TLR-7 agonist-induced lupus mouse by flow cytometry, PCR, and immunofluorescence (IF). CD4+ T cells from human peripheral blood were isolated to investigate the expression of NRP-1 in healthy control and the patients with SLE $(n=40)$.

Results: The frequency of NRP-1 positivity in CD4+ T cells in spleen was significantly higher in lupus mouse group (median [interquartile range]: 15.34 [14.84] $\%)$ compared to vehicle mouse group $(4.0[2.77] \%)$. The quantitative analysis of fluorescense intensity in kidney stained for NRP-1 revealed the increased level in lupus group compared to vehicle group. The CD4+ T cells from peripheral blood mononuclear cells in the patients with lupus also showed significantly higher frequency of NRP-1 positive CD4+ T cells than those from healthy controls. Comparing the correlation of the expression of NRP-1 and disease activity with SLEDAI, C3, C4, and anti-DNA antibodies, the significant correlation between NRP-1 and disease activity markers were confirmed.

Conclusion: Our results demonstrate that higher expression of NRP-1 in CD4+ $T$ cells and its significant correlation with disease activity of SLE. These results indicate that pathologic contribution of NRP-1 in the pathogenesis of SLE and potential of targeting NRP-1 for the treatment of SLE.

\section{REFERENCES:}

[1] Nishide M, Kumanogoh A. The role of semaphorins in immune responses and autoimmune rheumatic diseases. Nat Rev Rheumatol. 2018 Jan;14(1):19-31.

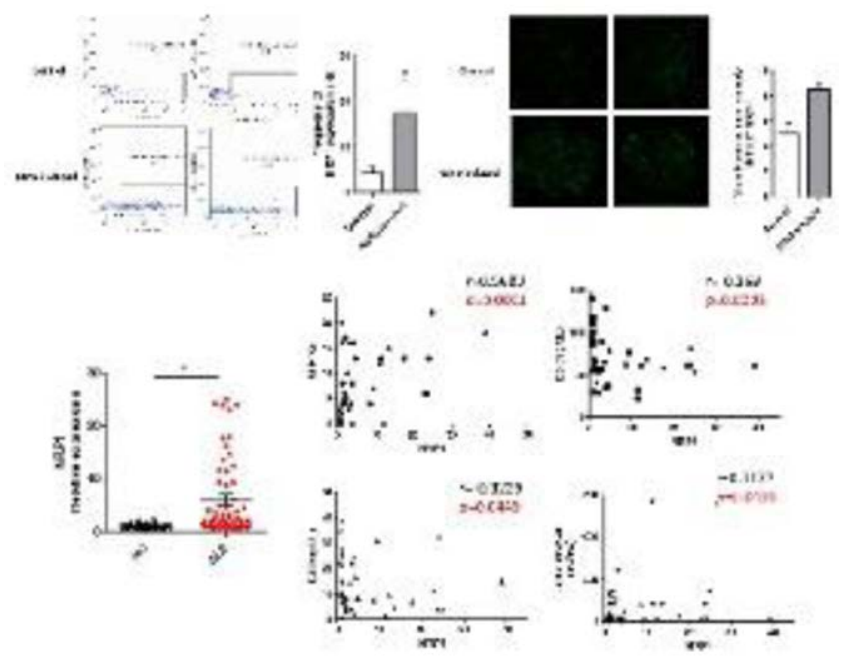

Disclosure of Interests: None declared

DOI: 10.1136/annrheumdis-2021-eular.3388

\section{$\mathrm{AB} 0085$ \\ ANTI-MULLERIAN HORMONE IN SYSTEMIC LUPUS ERYTHEMATOSUS (SLE)}

Z. Alekberova ${ }^{1}$, R. Goloeva ${ }^{1}$, M. Cherkasova ${ }^{1}$, A. Lila ${ }^{1}{ }^{1}$ VA Nasonova Research Institute of Rheumatology, Systemic Rheumatic Diseases, Moscow, Russian Federation

Background: Anti-Mullerian hormone $(\mathrm{AMH})$ is one of the key parameters for assessing reproductive function and ovarian reserve. The level of $\mathrm{AMH}$ correlates with the residual follicular pool in women of reproductive age.
Objectives: To assess AMH levels in SLE female patients of child-bearing potential, and analyze the relationship between AMH levels and disease severity, as well as relationship between serum AMH levels and different therapeutic regimens. Methods: The study group included 12 women with SLE aged 20-40 years, and the control group - 15 age-matching healthy women. SLE diagnosis was established based on the EULAR/ACR classification criteria, 2019. The SLEDAI 2K was used to evaluate disease activity, and the SLICC index - to evaluate damage. AMH levels was measured using ELISA. AMH reference values ranged within 1.0-10.6ng / $\mathrm{ml}$. Values $<1.0$ were interpreted as a decreased ovarian reserve.

Results: SLE patients' mean age was 33.5 [29;38] years, and control group subjects' - $32.0[26 ; 35]$ years. Mean disease duration was 11.5 [2.8;18] years. Acute, subacute and chronic SLE was established in $33 \%, 17 \%$, and $50 \%$, respectively. Disease activity according to SLEDAI $2 \mathrm{~K}$ was low in $25 \%$, moderate - in $33 \%$, and high - in $42 \%$ of pts. The SLICC damage baseline evaluation: no damage $-17 \%$, low $-50 \%$, medium $-16.5 \%$, high- $16.5 \%$.

Mean AMH level was $3.5 \mathrm{ng} / \mathrm{ml}$ in SLE pts, and $3.1 \mathrm{ng} / \mathrm{ml}$ - in the control group showing no statistical difference. A decrease in ovarian reserve (AMH less than $1.0 \mathrm{ng} / \mathrm{ml}$ ) was significantly more common in SLE pts - $42 \%$ vs $13 \%$ in the control group. No correlation was found between AMH levels and the SLICC damage index, $\mathrm{AMH}$ and SLEDAI $2 \mathrm{~K}$, as well as AMH and SLE duration or clinical course. Detailed analysis showed that all pts with reduced ovarian function had $\mathrm{CP}$ included into their therapeutic regimens; the only exclusion within this subgroup i.e., normal AMH level- $6 \mathrm{ng} / \mathrm{ml}$ and preserved ovarian function - was documented in a patient who received a cumulative $\mathrm{CP}$ dose of $5.6 \mathrm{~g}$. In all other patients a cumulative CP dose was higher, i.e.: $7.2 \mathrm{~g}, 7.8 \mathrm{~g}, 10.6 \mathrm{~g}$, and $18.4 \mathrm{~g}-1$ patient per each dose value. Three pts with low AMH levels within $0.1-0.3 \mathrm{ng} / \mathrm{ml}$ were 39 years old, while AMH reference values in 33-39y age group are $0.77-5.24 \mathrm{ng} / \mathrm{ml}$. Analysis of gynecological history indicate that episodes of menstrual disorders were significantly more often reported in SLE pts $(50 \%$ vs $20 \%$ in controls, $p=0.001$ ), similarly, gynecological diseases were also documented in $50 \%$ of SLE pts (chronic salpyngo-oophoritis, colpitis, endometriosis and uterine endometrioma, subserous uterine myoma, cervical dysplasia, cervical erosion), meanwhile low AMH was found only in 4 SLE pts; there was only 1 subject with gynecological condition - teratoma of the ovaries - in the control group (favorable outcome - surgical removal, preserved fertility and two births after surgery). Conclusion: Ovarian reserve was significantly more often reduced in women with SLE compared to healthy controls. CP therapy negatively affects ovarian function, and most likely in a dose-dependent manner. In this study, 4 out of 5 patients with reduced ovarian function had gynecological diseases additionally compromising the ovarian reserve. Further studies are needed to clarify potential contribution of both - autoimmune inflammation and SLE therapy - into development of gynecological diseases and loss of fertility.

Disclosure of Interests: None declared

DOI: 10.1136/annrheumdis-2021-eular.3402

\section{AB0086 PREGNANCY OUTCOMES IN SYSTEMIC LUPUS ERYTEMATOSUS (SLE)}

Z. Alekberova ${ }^{1}$, R. Goloeva ${ }^{1}$, T. Popkova ${ }^{1}{ }^{~}{ }^{2}$ V A Nasonova Research Institute of Rheumatology, systemic rheumatic disease, Moscow, Russian Federation

Background: There's no doubt that SLE has negative effect not only on the course of pregnancy, but also on maternal and fetal outcomes.

Objectives: To clarify pregnancy outcomes in patients with SLE by means of retrospective analysis.

Methods: The study group included 12 women with SLE aged 20-40 years, and the control group - 15 age-matching healthy women. SLE diagnosis was established based on the EULAR/ACR classification criteria, 2019. The SLEDAI $2 \mathrm{~K}$ was used to evaluate disease activity, and the SLICC index - to evaluate damage. Completed by all women issue-specific questionnaire covered clinical symptoms of SLE and details of obstetric history (characteristics of menstrual and reproductive function, presence of genital and extragenital pathology, previous pregnancies outcomes; previous intrauterine interventions, a history of coagulopathy).

Results: SLE patients' mean age was 33.5 [29;38] years, and control group subjects' - $32.0[26 ; 35]$ years. Mean disease duration was $11.5[2.8 ; 18]$ years. Acute subacute and chronic SLE was established in $33 \%, 17 \%$, and $50 \%$, respectively. Disease activity according to SLEDAI $2 \mathrm{~K}$ was low in $25 \%$, moderate - in $33 \%$, and high - in $42 \%$ of pts. The SLICC damage baseline evaluation: no damage $-17 \%$, low $-50 \%$, medium $-16.5 \%$, high- $16.5 \%$. Past pregnancies were documented in 7 out of 12 SLE patients and 6 healthy subjects from the control group. Spontaneous vaginal delivery of a full-term baby was documented in both groups. Unfavorable pregnancy outcomes were significantly more frequent in SLE pts ( $31 \%$ vs $13 \%$ in the control group, $p=0.001$ ). Two SLE pts after cyclophosphane therapy (10.6 and $18.4 \mathrm{~g}$ cumulative dose) and low AMH levels $(<1 \mathrm{ng} / \mathrm{ml})$ failed to become pregnant, although never used contraception. 